\title{
STING and liver disease
}

\author{
Can Chen $^{1} \cdot$ Rui-Xia Yang ${ }^{1} \cdot$ Hua-Guo $\mathrm{Xu}^{1}$ (D)
}

Received: 6 May 2021/Accepted: 18 June 2021/Published online: 23 June 2021

(C) Japanese Society of Gastroenterology 2021

\begin{abstract}
STING (stimulator of interferon genes) also known as transmembrane protein 173 (TMEM173) is a cytoplasmic DNA sensor which can be activated by the upstream cyclic dinucleotides (CDNs). This activation produces cytokines such as interferons and pro-inflammatory factors via the downstream IRF3 and NF- $\kappa \mathrm{B}$ pathways, triggering an innate immune response and adaptive immunity to maintain homeostasis. STING is mainly expressed and activated in non-parenchymal cells, thus exerting a corresponding effect to maintain the homeostasis of the liver. In viral hepatitis, interferons and pro-inflammatory factors produced after STING activation initiate the immune response to inhibit virus replication and assembly. In the case of metabolic diseases of the liver, the activation of STING in kupffer cells and hepatic stellate cells leads to inflammation, the proliferation of connective tissue, and metabolic disorders in the hepatocytes, promoting the occurrence and development of the disease. In hepatocellular carcinoma, STING has two contradictory roles. When STING is activated in dendritic cells and macrophages, a large number of cytokines can be produced to initiate innate immune effects directly and to exert adaptive immunity through the recruitment and activation of $\mathrm{T}$ cells; however, aberrant activation of the STING pathway leads to a weakening of immune function and promotes oncogenesis and metastasis. Here, we summarize the
\end{abstract}

Can Chen and Rui-Xia Yang have contributed equally to this work.

Hua-Guo Xu

huaguoxu@njmu.edu.cn

1 Department of Laboratory Medicine, The First Affiliated Hospital, Nanjing Medical University, 300 Guang Zhou Road, Nanjing 210029, Jiangsu Province, China interactions between STING and liver disease that have currently been identified and how to achieve therapeutic goals by modulating the activity of the STING pathway.

Keywords STING - Viral hepatitis - Non-alcoholic fatty liver disease $\cdot$ Liver fibrosis and cirrhosis - Hepatocellular carcinoma

\section{Molecular regulation of STING and its signaling pathways}

STING, also known as MITA, MPYS, ERIS, and TMEM173, is an endoplasmic reticulum (ER) associated dimeric protein that was discovered in 2008 [1-4]. STING consists of an N-terminal domain that spans the ER membrane four times, and a cytoplasmic C-terminal region, which contains the ligand-binding domain (LBD) and the C-terminal tail [5]. STING is mainly distributed in various tissues and organs, and its expression in tissue cells can be controlled by gene regulation. Hypermethylation of $\mathrm{CpG}$ land in the STING promoter interferes with STING transcription and downregulates STING expression levels [6]. Transcription factors CREB and c-Myc can combine with human STING (hSTING) promoter to enhance its transcription activity to increase the expression level of STING [7]. STING splicing isoform, including MITA-related protein (MRP), negatively downregulates STINGinduced interferon (IFN) production [8]. MicroRNAs bind to the 3'-untranslated region of hSTING to silence STING translation process and decrease the expression level of STING at the post-transcriptional level [9]. Post-translational modifications (PTMs) of STING can also modulate STING function. Palmitoylation at the STING Cysteine 
$88 / 91$ is critical for the recruitment and activation of TANK-binding kinase 1 (TBK1) and interferon regulatory factor 3 (IRF3) [10]. In addition to activating STINGTBK1 pathway, which elicits the release of IFNs to exert anti-viral effects, polyubiquitination of STING can also degrade STING and abrogate its effects [11]. TBK1 and I $\kappa$ B kinases (IKK) directly phosphorylate STING and then recruit IRF3 to produce IFNs [12].

In the cytoplasm, activation of STING is mainly through recognition and binding of exogenous cyclic dinucleotides (CDNs) such as cyclic diguanosine monophosphate (c-diGMP) and cyclic diadenylate monophosphate (c-di-AMP) produced by bacteria or endogenous CDNs including cyclic GMP-AMP (cGAMP). When ectopic DNA appears in the cytoplasm, including exogenous DNA produced by viruses and bacteria, and endogenous DNA such as nuclear DNA and mitochondrial DNA, cyclic GMP-AMP synthase (cGAS) as a DNA sensor can detect and bind to cytoplasmic DNA. Subsequently, cGAS is converted to cGAMP, which binds and activates STING [13]. Some RNA viruses trigger the release of cytoplasmic mtDNA to activate cGAS-STING signaling pathway [14]. Upon binding of CDNs, STING undergoes a conformational change and transfers from the ER to the Golgi [15]. Subsequently, STING recruits and activates TBK1 and IRF3, which translocate to the nucleus for transcriptional production of IFNs [1]. STING can also interact with IKK complexes, including IKK $\alpha$, IKK $\beta$, and IKKe. Among them, IKK $\varepsilon$ can synergistically phosphorylate IRF3 with TBK1, while IKK $\beta$ and IKK $\alpha$ activate NF- $\kappa B$ [12]. Activated NF- $\kappa B$ dimers enter nucleus to transcribe proinflammatory factors, including IL- $1 \beta$, IL- 6 , and TNF- $\alpha$ [12]. Cytokines such as IFNs and pro-inflammatory factors initiate innate immune responses to eliminate intracellular pathogens, and activate antigen-presenting cells (APCs) to cross-presentation antigens to $\mathrm{T}$ cells, thereby triggering an adaptive $\mathrm{T}$-cell immune response [1, 12]. In addition, STING activation can induce non-immunological reactions such as cell autophagy, senescence, apoptosis, and necrosis [16] (Fig. 1).

STING is not homogeneously distributed in liver. Compared to hepatocytes, STING is mainly expressed and activated in hepatic non-parenchymal cells (NPCs), including Kupffer cells, sinusoidal endothelial cells, and hepatic stellate cells (HSCs). Exogenous pathogens mainly infect hepatocytes, so the effects of STING in liver are mainly exerted through cellular cross-talking [17].

This review summarizes the current interaction between STING and liver diseases and how to exogenously regulate STING activity to achieve therapeutic effects.

\section{Viral hepatitis type B}

HBV is an enveloped double-stranded DNA virus and HBV genomic DNA is a relaxed circular double-stranded DNA molecule (rcDNA). After HBV invades hepatocytes, HBV genome enters the nucleus, at which point rcDNA is extended and converted into covalently closed circular DNA (ccDNA). HBV uses cccDNA as a template to transcribe four mRNA. The longest $3.5 \mathrm{~kb}$ fragment is called pre-genomic RNA (pgRNA), carrying all genetic information. The pgRNA is used as a template to generate offspring rcDNA to form a new HBV [18].

Currently, it has been shown that STING activation can produce IFNs to inhibit HBV replication [19-24]. The polymerase polymorphism rt269I in HBV genotype $\mathrm{C}$ and telomerase-derived 16-mer peptide GV1001 cause mitochondrial stress and release mtDNA in human HCC cell line HepG2, which activates STING-TBK1-IRF3 pathway and produces IFN- $\beta$ to exert anti-viral effects $[19,20]$. However, STING mainly activates downstream TBK1IRF3 pathway, whereas MRP only activates IKK-NF- $\mathrm{BB}$ pathway to inhibit HBV replication [22]. Besides, ISG56 produced by cGAS-STING pathway in human hepatoma Li23 cell inhibits viral assembly without influencing HBV DNA expression [25].

Although STING can inhibit HBV replication and assembly, HBV still causes persistent liver infection. In terms of $\mathrm{HBV}$ itself, when $\mathrm{HBV}$ invades human hepatoma HepG2-hNTCP cells, little immunostimulatory HBV DNA is produced, and HBV genome is packaged by viral capsids to escape cGAS recognition [26, 27]. In addition, several studies have shown that HBV can inhibit STING mRNA expression [27, 28]. The anti-viral effect of K63-linked STING polyubiquitination is diminished after STING binds to the RT and RH sites on HBV DNA polymerase [17, 29].

Currently, to address the low expression of STING in hepatocytes, we can exogenously introduce STING plasmids into hepatocytes or apply STING agonists [17, 30]. The treatment of HBV with anti-viral drugs can be accompanied by the use of methylation inhibitors to inhibit STING promoter methylation, thus improving the therapeutic effect [31]. The latest findings show that manganese $\left(\mathrm{Mn}^{2+}\right)$ promotes the production of type I interferon and inhibits HBV replication by enhancing the affinity between STING and cGAMP [32].

\section{Viral hepatitis type $\mathrm{C}$}

$\mathrm{HCV}$ is a single positive-stranded RNA virus and $\mathrm{HCV}$ genome is composed of non-coding regions (NCR) at the 5 '-terminal and 3'-terminal and an open-reading frame 


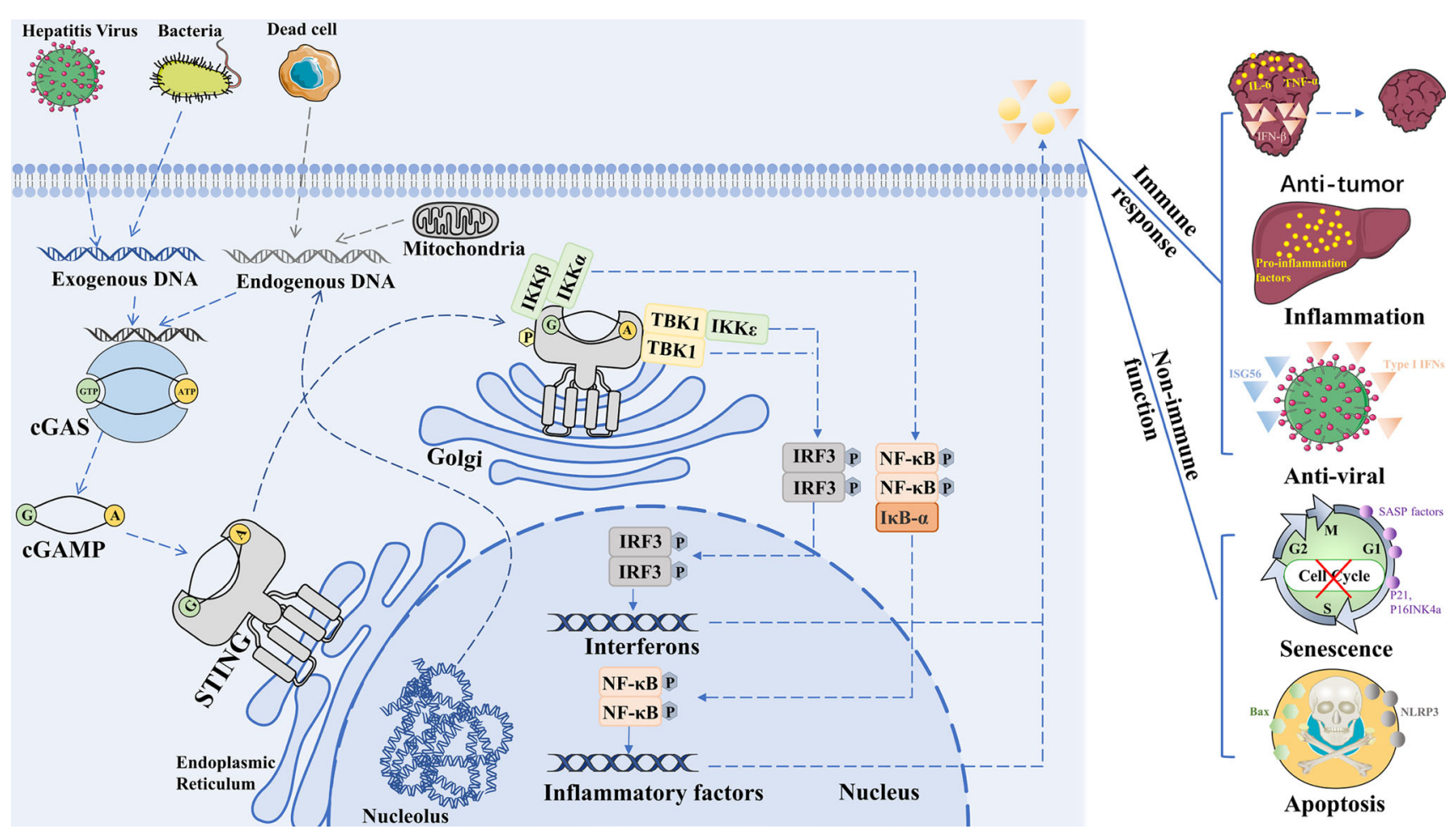

Fig. 1 cGAS-STING signaling pathway in liver immune cells. When exogenous pathogens such as viruses and bacteria invade cells, the released exogenous DNA and endogenous host DNA are recognized by cGAS and activate STING through the production of cGAMP. The activated STING produces a large number of cytokines such as

(ORF) in the middle. Starting from the 5'-terminal of the ORF, the coding region consists of seven gene regions, including NS3 and NS4 [33].

Yi et al. found that STING not only generates type I IFNs and ISGs through TBK1-IRF3 pathway to initiate anti-viral responses, but also silences HCV 1b/Con1 replicon replication in human hepatoma Huh7.5 cells [34].

Similarly, HCV can inhibit STING and its pathway. Several studies have shown that the HCV NS4B protein can interact directly with STING because of its N-terminal structure similar to that of STING. This interaction disrupts the interaction of STING with mitochondrial anti-viral signal (MAVS), to attenuate retinoic acid-inducible gene I (RIG-I)-induced IFN- $\beta$ production in Huh7.5 cells [35]. Since both are located in the ER, the interaction of $\mathrm{HCV}$ NS4B and STING limits the positional transition of STING to disrupt the interaction between STING and TBK1 in PH5CH8 cell (an immortalized human hepatocytes cell line) [36]. In the above process, NS3/A can collaborate with NS4B to inhibit STING activity and IFNs production $[35,36]$. Further researches reveal that, unlike HCV genotype $1 \mathrm{~b}$ Con 1 replicon, STING has no inhibitory effect on HCV 2A/JFH1 replicon. However, HCV 2A NS4B, interferons and inflammatory factors mainly through the downstream TBK1-IRF3 and IKK-NF- $\kappa B$ signaling pathways. After these cytokines are released extracellularly, they can exert immunological effects such as anti-cancer, anti-viral, inflammation, and nonimmunological effects such as cell senescence and apoptosis

especially in the B region, can inhibit STING aggregation and attenuates STING-mediated anti-viral effects [34].

Currently, it has been found that inoculation of recombinant baculovirus into $\mathrm{HCV}$-infected hepatocytes reactivates STING and initiates STING-mediated immune responses [37].

\section{Non-alcoholic fatty liver disease}

Non-alcoholic fatty liver disease (NAFLD) is a metabolic stress liver injury associated with insulin resistance and genetic susceptibility, including simple fatty liver, non-alcoholic steatohepatitis (NASH), and its associated cirrhosis and liver cancer [38].

It has been found that NASH patients have an abnormal liver mitochondrial function and significantly higher cytoplasmic mtDNA content than normal [39]. When hepatic phagocytes engulf apoptotic or dead hepatocytes, their selfDNA enters the cytoplasm and then activates cGASSTING pathway [40]. Besides, excessive deposition of lipids in NAFLD patient liver leads to oxidative stress damage to ER, thus activating STING-TBK1 pathway and promoting hepatic inflammation [41]. 
Most studies have found that activation of STINGTBK1-IRF3 and IKK-NF- $\kappa$ B pathways in hepatic phagocytes from high-fat diet (HFD) feed wild-type mice (STING ${ }^{+}$, C57BL/6J background) produces cytokines, including IFNs, inflammatory factors, $\alpha$ smooth muscle actin (aSMA), TGF- $\beta$, and type IA collagen A1 (Col1a1). Of these, IFNs and inflammatory factors mainly act on mouse hepatocytes, causing abnormal inflammation in liver [42-45]; whereas aSMA, TGF- $\beta$, and Colla1 mainly activate HSCs and exacerbate liver fibrosis through paracrine secretion [42, 43, 45]. Besides, activation of STINGTBK1 pathway in HFD-feed WT mouse liver not only causes hepatic metabolic disturbances, including insulin resistance and lipid deposition in hepatocytes [42, 44], but also increases P62 phosphorylation levels and deposits insoluble P62 protein inclusions, which play an important pathogenic role in the formation of NASH [46]. However, some scholars have questioned whether these findings obtained from mice are suitable for humans, so further studies and elaborations are warranted [47].

\section{Liver fibrosis and cirrhosis}

Hepatitis virus, alcohol, and autoimmune factors can contribute to the development of liver fibrosis, which then progresses to cirrhosis. Certain pathogenic factors, such as viruses and fat deposits, can activate STING and STINGTBK1-mediated inflammatory responses [48]. Through cellular interactions and signal transduction, inflammation further activates HSCs and converts them into myofibroblast-like cells, which secret $\alpha$-SMA and hepatocyte growth factors to accelerate the formation of hepatic fibrosis [42, 43]. Besides, in CCl4-treated WT mouse hepatocytes, ER stress caused extensive phosphorylation of TBK1 and IRF3 through STING. At this time, mouse hepatocytes apoptosis occurred and the degree of hepatic fibrosis increased. This phenomenon had not been observed in STING-deficient mice [49]. Therefore, STING-mediated liver inflammation and hepatocyte death are the two main direct drivers of liver fibrosis.

\section{Hepatocellular carcinoma}

Hepatocellular carcinoma (HCC) is the most common subtype of primary liver cancer with a high mortality rate [50]. Unlike normal cells, the cytoplasm of hepatocellular carcinoma cells contains large amounts of ectopic DNA, such as tumor-derived DNA, mtDNA, and nuclear chromosome fragments [51]. When cytoplasmic DNA is recognized by cGAS in DCs and macrophages, STINGTBK1-IRF3 and IKK-NF- $\kappa$ B pathways are activated to produce IFNs, pro-inflammatory factors, and chemokines to restrict tumor cells proliferation. These cytokines also recruit DCs and NK cells around tumor tissues, forming a tumor-suppressive microenvironment infiltrated by tumorspecific lymphocytes and acting as a front-line defense against tumor immunity [52]. Besides, IFN- $\beta$ not only enhances the terminal differentiation of DCs and accelerates the maturation of DCs, promoting the cross-presentation of tumor-specific antigenic peptides from DCs to MHC class I molecules in CD8 $+\mathrm{T}$ cells to activate it [53], but also increases CXCL9, CXCL10, and other chemokine expression, which in turn induces T-lymphocytes to metastasize to tumor tissues, killing tumor cells and initiating adaptive immune responses [54]. Studies have found that when cGAS-STING pathway is activated in senescent liver fibroblasts or epithelial cells, large amounts of proinflammatory factors, chemokines, growth factors, and proteases are secreted via STING-IKK-NF- $\mathrm{B}$ signaling pathway, which is a typical feature of cellular senescence, also known as SASP [55]. SASP can stimulate inflammation in surrounding tumor tissue through paracrine and autocrine forms, accelerating senescence of cancer and precancerous cells, and also recruits NK cells and neutrophils to clear cancerous tissue $[56,57]$. Besides, STING activation produces non-immune functions, including autophagy, apoptosis, and necrosis, which effectively remove exogenous pathogens and cancer DNA and also facilitate antigen presentation to $\mathrm{T}$ cells to mediate $\mathrm{T}$-cell immune responses [58].

Conversely, STING signaling pathway promotes tumorigenesis and progression. IFN- $\beta$ generated by STING-TBK1-IRF3 pathway stimulates the production of immune checkpoint molecules, such as programmed cell death ligand 1 (PD-L1) and cytotoxic T-lymphocyte-associated protein 4 (CTLA-4), which bind to T-cell surface receptors to inhibit $\mathrm{T}$-cell activation, resulting in immune evasion [59, 60]. Besides, type I IFNs can induce persistent DNA damage during treatment with chemotherapy and radiotherapy techniques, which in turn aberrantly activate STING and cause long-term inflammation [61]. The latter causes tissue destruction and immunosuppression, decelerating cancer cell senescence and immortalizing cancer cells [57]. SASP secreted by HSCs was found to promote the formation of obesity-associated HCC [62]. Besides, STING-induced apoptosis of T and B lymphocytes impairs cellular immune functions [63].

For the treatment of HCC, Huang et al. found that tumor-targeted lipid-dendritic calcium phosphate nanoparticles (TT-LDCP) activate STING-TBK1-IRF3 pathway and increase the expression of IFNs, pro-inflammatory factors, and chemokines, causing DCs' aggregation into tumor microenvironment to increase CD8 $+\mathrm{T}$-cell infiltration and activation and inhibit HCC progression 
[64]. STING agonists can be used in combination with immune checkpoint inhibitors (ICIs). T-lymphocytes can be activated and infiltrate tumor tissue using STING agonists. At this point, ICIs, such as anti-PD-L1 and antiCTLA-4 antibodies, restore T-cell immune function to consistently suppress tumor growth and metastasis $[65,66]$. Besides, STING can be used in combination with chemotherapy and radiotherapy. Ionizing radiation and drug factors damage the genome of tumor cells, which in turn releases tumor dsDNA and activates cGAS-STING pathway in hepatic NPCs [67].

\section{Other liver diseases}

Alcoholic liver disease is mainly caused by long-term heavy alcohol consumption. In the early stages of alcoholic liver injury in WT $\mathrm{STING}^{+} \mathrm{C} 57 \mathrm{BL} / 6 \mathrm{~J}$ mice, alcohol induces hepatic ER stress via cytochrome P450, which then activates STING-TBK1-IRF3 pathway. Phosphorylated IRF3 binds to the intracellular pro-apoptotic molecule B-cell lymphoma 2-associated $\mathrm{X}$ protein and transits into mitochondria, initiating intracellular apoptotic signaling that causes apoptosis of hepatocytes. This apoptotic process is not associated with type I IFNs or inflammation. Differently, in the late stage of alcoholic liver disease, STING-TBK1 pathway activation is mainly through the recognition of damaged hepatocyte nuclear DNA by cGAS [68].

Liver dysfunction and radiation-induced liver disease occur during radiation therapy for HCC. Ionizing radiation causes apoptosis and necrosis in WT mouse hepatocytes, and hepatocyte self-DNA escapes and accumulates in the hepatic blood sinusoids. cGAS-STING pathway in hepatic NPCs recognizes and binds ectopic DNA in the blood sinusoids, producing type I IFNs and pro-inflammatory factors. Type I IFNs down-regulate the levels of superoxide dismutases (SODs) and increase the expression of reactive oxygen species (ROS) in hepatocytes, causing intracellular oxidative stress and sustained damage. Besides, type I IFNs induce the expression of CXCL10 and IFIT1 in hepatocytes, further aggravating mouse liver injury [69].

In addition to the above chemical liver injury, hepatic ischemia-reperfusion (IR) injury is also associated with STING. MicroRNA-24-3p was found to bind STING UTR to down-regulate the expression level of STING and inhibit phosphorylation of IRF3. In this process, the release of inflammatory factors was reduced and hepatic IR injury was alleviated, laterally suggesting that STING can promote the occurrence of hepatic ischemia-reperfusion injury in male mice [9]. Similarly, Wang et al. found that hepatic IR injury was also closely related to STING-NLRP3 axis. Following liver post-IR injury, aged mice hepatocytes release mtDNA, which activates cGAS-STING pathway in hepatic macrophages to produce inflammatory factors and chemokines, including nuclear binding domain and leucine-rich repeat protein 3 (NLRP3). Increased activation of NLRP3 further upregulates inflammatory factor expression levels to exacerbate liver IR injury [70].

\section{STING agonists and antagonists}

STING agonists include natural CDNs, synthetic CDN agonists, and non-cyclic dinucleotide molecules. As ligands for STING, CDNs and CDN analogs such as 3'3'cAIMP can directly bind the C-terminus of STING $[71,72]$. In addition to the above two categories, there are several non-CDN small-molecule STING agonists, including DMXAA, CMA, DSDP, G10, and BNBC. DMXAA and CMA can bind directly to the mSTING C-terminal domain to activate STING and thus exert antiviral effects [73, 74]; while G10 promotes phosphorylation of hSTING [75]. Besides, further studies are needed to elucidate how DSDP and BNBC activate STING [76, 77].

In the liver, $\mathrm{CDN}$ agonists and synthetic $\mathrm{CDN}$ agonists such as 3'3'-cAIMP, cGAMP, and c-di-AMP enhance the cross-talk between hepatocytes and immune cells. IFNs, ISGs, and chemokines produced after the activation of STING signaling pathway in immune cells can induce hepatocyte apoptosis and autophagy, and initiate immune responses to inhibit viral infection and hepatocarcinogenesis [71, 78, 79]. Differently, CMA and DMXAA can directly activate mSTING in hepatic macrophages to increase IFNs and pro-inflammatory expression, ultimately causing hepatic steatosis and inflammation [43, 80, 81] (Fig. 2a; Table 1).

In the application of STING inhibitors, STIM1, as a calcium sensor, was found to attach STING to ER and limit position change after STING activation [82]. C-178 and C-176 can covalently bind to Cys91 on mSTING and reduce the palmitoylation of $\mathrm{mSTING}$, with $\mathrm{C}-178$ mainly inhibiting ISGs expression and C- 176 predominantly reducing IFN-I expression. Similarly, H-151 irreversibly binds to Cys91 in hSTING to inhibit hSTING palmitoylation [83]. Currently, these STING agonists are used to inhibit the activation of STING and reduce the expression of IFNs and inflammatory factors to alleviate autoimmune diseases and auto-inflammatory diseases. RDV inhibits the secretion of inflammatory factors and attenuates liver inflammation by significantly eliminating the phosphorylation of STING and its downstream IRF3 and NF- $\mathrm{NB}$ pathways [84] (Fig. 2b; Table 1). 
Fig. 2 The different effects of using STING agonists and inhibitors in the liver. In the case of STING agonists, they mainly exert anti-viral and anticancer effects through the IFN produced, while for antagonists, they are currently mainly used to alleviate liver inflammation
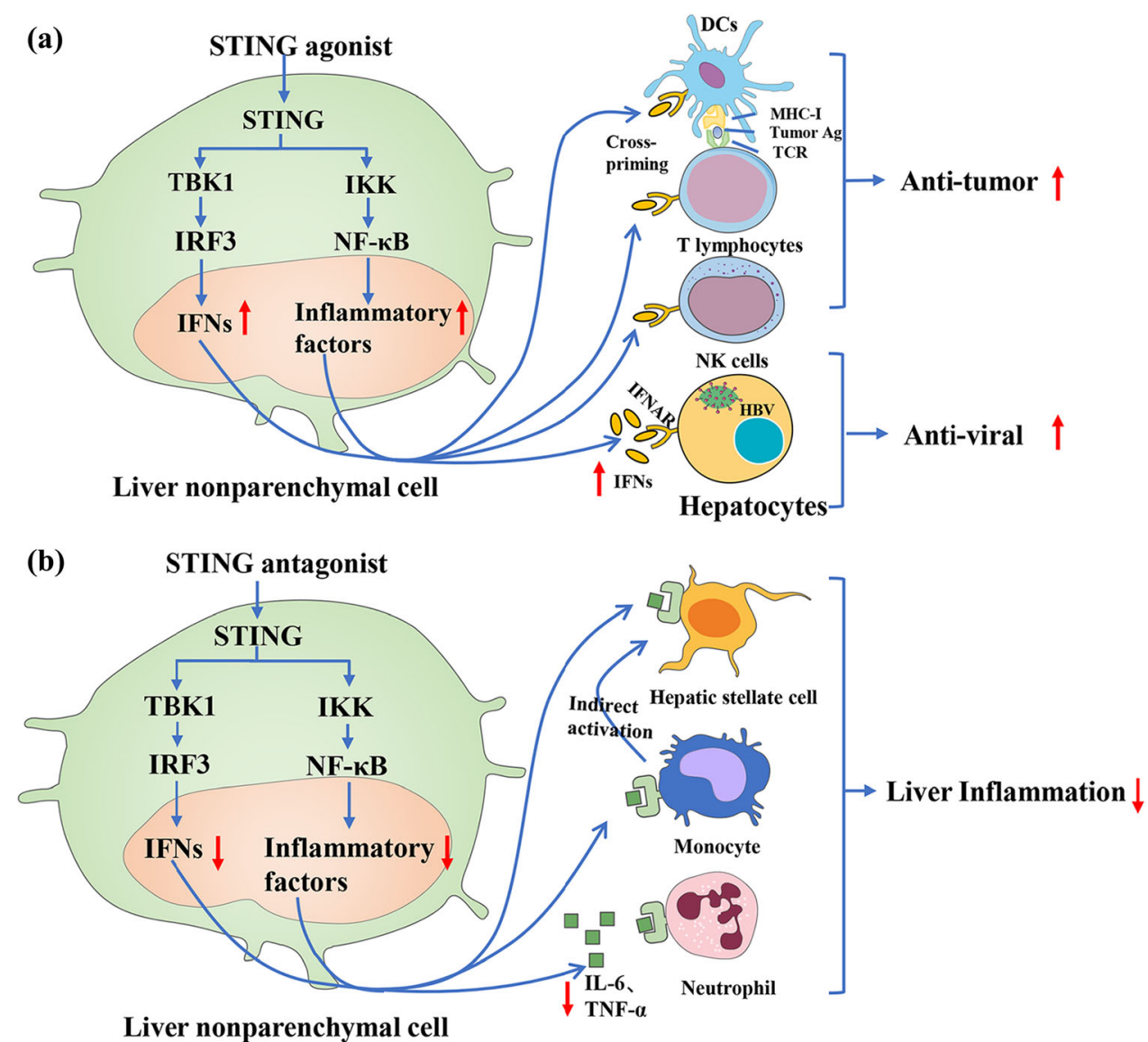

Table 1 Overview of STING agonists and antagonists in liver disease

\begin{tabular}{|c|c|c|c|c|c|}
\hline Type & & $\begin{array}{l}\text { Related to } \\
\text { hSTING }\end{array}$ & Ways to activate/inhibit STING & Effects on liver disease & References \\
\hline \multirow[t]{5}{*}{ Agonists } & c-di-AMP & Yes & It directly binds the CTD of STING & It can resist $\mathrm{HCV}$ infection & [79] \\
\hline & $\begin{array}{l}\text { 2’3'- } \\
\text { cGAMP }\end{array}$ & Yes & $\begin{array}{l}\text { It has a greater affinity than cGAMP to bind to } \\
\text { STING }\end{array}$ & $\begin{array}{l}\text { It can inhibit hepatitis virus infection } \\
\text { and alleviate HCC }\end{array}$ & [78] \\
\hline & $\begin{array}{l}\text { 3'3'- } \\
\text { cAIMP }\end{array}$ & Yes & $\begin{array}{l}\text { It has stronger binding affinity to STING and can } \\
\text { activate all known human STING alleles }\end{array}$ & $\begin{array}{l}\text { It can reduce tumor burden and induce } \\
\text { liver inflammation }\end{array}$ & {$[71]$} \\
\hline & DMXAA & No & It directly binds the CTD of STING & $\begin{array}{l}\text { It inhibits HBV replication and } \\
\text { aggravates hepatic steatosis and } \\
\text { inflammation }\end{array}$ & {$[74]$} \\
\hline & CMA & No & It directly binds the CTD of STING & $\begin{array}{l}\text { It inhibits HBV replication and } \\
\text { aggravates hepatic steatosis and } \\
\text { inflammation }\end{array}$ & {$[73]$} \\
\hline Antagonist & Remdesivir & Yes & $\begin{array}{l}\text { It can block STING/IRF3 signaling, and the } \\
\text { specific mechanism is unknown }\end{array}$ & $\begin{array}{l}\text { It attenuates high-fat diet-induced } \\
\text { NAFLD }\end{array}$ & {$[84]$} \\
\hline
\end{tabular}

STING stimulator of interferon genes, $c$-di-AMP cyclic diadenylate monophosphate, $c$ GAMP cyclic GMP-AMP, $c A I M P$ cyclic AMP-IMP, $C M A$ 10-carboxymethyl-9-acridanone, DMXAA 5,6-dimethylxanthenone-4-acetic acid

\section{Conclusion}

The effects induced by the activation of STING and its pathway are critical in liver diseases. Although activation of cGAS-STING pathway can resist the invasion of exogenous pathogens in the liver, excessive or abnormal activation of this pathway is detrimental. The ability to treat and prevent liver diseases through the regulation of STING expression is an area that deserves further investigation. MiRNAs inhibit the translation of STING and 
reduce its expression level, so whether the exogenous introduction of synthetic miRNAs in hepatocytes can achieve therapeutic effects still needs further investigation. In addition to the identified modulation of STING promoter hypermethylation to treat HBV infection, further analysis is needed to determine whether modulation of STING expression and function by other PTMs can achieve the desired therapeutic effect. Another research area is to further investigate the application of STING agonists and inhibitors in liver diseases. cGAS-STING pathway is a double-edged sword that can be activated or inhibited to achieve the desired effect. For example, in hepatitis virus infection and cancer, stimulation of cGAS-STING pathway can prevent further damage by viral and tumor cells. In contrast, in metabolic liver injury, abnormal activation can lead to exacerbation of the disease. Since liver diseases are not a single existence for progressive occurrence and development, consideration should be given to whether the side effects that arise when activating or inhibiting the pathway interfere with the desired therapeutic effect. Therefore, the use of STING as a target for the treatment of liver disease is of high research value.

Acknowledgements This study was supported by the Natural Science Foundation of Jiangsu Province of China (BK20181492), the National Key Clinical Department of Laboratory Medicine of China in Nanjing, Key laboratory for Laboratory Medicine of Jiangsu Province (ZDXKB2016005), and by the Priority Academic Program Development of Jiangsu Higher Education Institutions.

Author contributions All authors contributed to the draft and have seen and approved the final version of the report.

Funding The funder of the study had no role in the study design, data collection, data analysis, data interpretation, or writing of the manuscript. The corresponding author had full access to all the data in the study and has final responsibility for the decision to submit for publication.

\section{Declarations}

Conflict of interest The authors declare that they have no conflict of interest.

\section{References}

1. Ishikawa H, Barber GN. STING is an endoplasmic reticulum adaptor that facilitates innate immune signalling. Nature. 2008;455:674-8.

2. Sun W, Li Y, Chen L, et al. ERIS, an endoplasmic reticulum IFN stimulator, activates innate immune signaling through dimerization. Proc Natl Acad Sci USA. 2009;106:8653-8.

3. Zhong B, Yang Y, Li S, et al. The adaptor protein MITA links virus-sensing receptors to IRF3 transcription factor activation. Immunity. 2008;29:538-50.

4. Jin L, Waterman PM, Jonscher KR, et al. MPYS, a novel membrane tetraspanner, is associated with major histocompatibility complex class II and mediates transduction of apoptotic signals. Mol Cell Biol. 2008;28:5014-26.

5. Gao P, Ascano M, Zillinger T, et al. Structure-function analysis of STING activation by $\mathrm{c}\left[\mathrm{G}\left(2^{\prime}, 5^{\prime}\right) \mathrm{pA}\left(3^{\prime}, 5^{\prime}\right) \mathrm{p}\right]$ and targeting by antiviral DMXAA. Cell. 2013;154:748-62.

6. An X, Zhu Y, Zheng T, et al. An analysis of the expression and association with immune cell infiltration of the cGAS/STING pathway in pan-cancer. Mol Ther Nucleic Acids. 2019;14:80-9.

7. Wang YY, Jin R, Zhou GP, et al. Mechanisms of transcriptional activation of the stimulator of interferon genes by transcription factors CREB and c-Myc. Oncotarget. 2016;7:85049-57.

8. Rodríguez-García E, Olagüe C, Ríus-Rocabert $\mathrm{S}$, et al. TMEM173 alternative spliced isoforms modulate viral replication through the STING pathway. Immunohorizons. 2018;2:363-76.

9. Shen A, Zheng D, Luo Y, et al. MicroRNA-24-3p alleviates hepatic ischemia and reperfusion injury in mice through the repression of STING signaling. Biochem Biophys Res Commun. 2020;522:47-52.

10. Mukai K, Konno H, Akiba T, et al. Activation of STING requires palmitoylation at the Golgi. Nat Commun. 2016;7:11932.

11. Wang Q, Liu X, Cui Y, et al. The E3 ubiquitin ligase AMFR and INSIG1 bridge the activation of TBK1 kinase by modifying the adaptor STING. Immunity. 2014;41:919-33.

12. Abe T, Barber GN. Cytosolic-DNA-mediated, STING-dependent proinflammatory gene induction necessitates canonical NF- $\kappa \mathrm{B}$ activation through TBK1. J Virol. 2014;88:5328-41.

13. Ablasser A, Goldeck M, Cavlar T, et al. cGAS produces a 2'-5'linked cyclic dinucleotide second messenger that activates STING. Nature. 2013;498:380-4.

14. Aguirre S, Luthra P, Sanchez-Aparicio MT, et al. Dengue virus NS2B protein targets cGAS for degradation and prevents mitochondrial DNA sensing during infection. Nat Microbiol. 2017;2:17037.

15. Ishikawa H, Ma Z, Barber GN. STING regulates intracellular DNA-mediated, type I interferon-dependent innate immunity. Nature. 2009;461:788-92.

16. Liu S, Guan W. STING signaling promotes apoptosis, necrosis, and cell death: an overview and update. Mediat Inflamm. 2018;2018:1202797.

17. Thomsen MK, Nandakumar R, Stadler D, et al. Lack of immunological DNA sensing in hepatocytes facilitates hepatitis B virus infection. Hepatology. 2016;64:746-59.

18. Seeger C, Ganem D, Varmus HE. Biochemical and genetic evidence for the hepatitis B virus replication strategy. Science. 1986;232:477-84.

19. Lee SY, Choi YM, Oh SJ, et al. rt269I type of hepatitis B virus (HBV) leads to HBV e antigen negative infections and liver disease progression via mitochondrial stress mediated type I interferon production in chronic patients with genotype $\mathrm{C}$ infections. Front Immunol. 2019;10:1735.

20. Choi YM, Kim H, Lee SA, et al. A telomerase-derived peptide exerts an anti-hepatitis B virus effect via mitochondrial DNA stress-dependent type I interferon production. Front Immunol. 2020;11:652.

21. Dansako H, Imai H, Ueda $Y$, et al. High-level expression of STING restricts susceptibility to HBV by mediating type III IFN induction. FASEB Bioadv. 2019;1:67-80.

22. Liu S, Zhao K, Su X, et al. MITA/STING and its alternative splicing isoform MRP restrict hepatitis B virus replication. PLoS ONE. 2017; 12:e169701.

23. He J, Hao R, Liu D, et al. Inhibition of hepatitis B virus replication by activation of the cGAS-STING pathway. J Gen Virol. 2016;97:3368-78.

24. Guo F, Tang L, Shu S, et al. Activation of Stimulator of Interferon Genes in Hepatocytes Suppresses the Replication of 
Hepatitis B Virus. Antimicrob Agents Chemother. 2017;61:e00771.

25. Dansako H, Ueda Y, Okumura N, et al. The cyclic GMP-AMP synthetase-STING signaling pathway is required for both the innate immune response against $\mathrm{HBV}$ and the suppression of HBV assembly. Febs j. 2016;283:144-56.

26. Lauterbach-Rivière L, Bergez $M$, Mönch $S$, et al. Hepatitis $B$ virus DNA is a substrate for the cGAS/STING pathway but is not sensed in infected hepatocytes. Viruses. 2020;12:592.

27. Verrier ER, Yim SA, Heydmann L, et al. Hepatitis B virus evasion from cyclic guanosine monophosphate-adenosine monophosphate synthase sensing in human hepatocytes. Hepatology. 2018;68:1695-709.

28. Karimi-Googheri M, Daneshvar H, Khaleghinia M, et al. Decreased expressions of STING but not IRF3 molecules in chronic HBV infected patients. Arch Iran Med. 2015;18:351-4.

29. Liu Y, Li J, Chen J, et al. Hepatitis B virus polymerase disrupts K63-linked ubiquitination of STING to block innate cytosolic DNA-sensing pathways. J Virol. 2015;89:2287-300.

30. Alexopoulou A, Vasilieva L, Karayiannis P. New approaches to the treatment of chronic hepatitis B. J Clin Med. 2020;9:3187.

31. Wu CS, Zhao Q, Zhang J, et al. Methylation status of the stimulator of interferon genes promoter in patients with chronic hepatitis B. Medicine (Baltimore). 2018;97:e13904.

32. Lin M, Guo R, Ma C, et al. Manganese breaks the immune tolerance of HBs-Ag. Open Forum Infect Dis. 2021;8:oab028.

33. Lindenbach BD, Rice CM. Unravelling hepatitis C virus replication from genome to function. Nature. 2005;436:933-8.

34. Yi G, Wen Y, Shu C, et al. Hepatitis C virus NS4B can suppress STING accumulation to evade innate immune responses. J Virol. 2016;90:254-65.

35. Nitta S, Sakamoto N, Nakagawa M, et al. Hepatitis C virus NS4B protein targets STING and abrogates RIG-I-mediated type I interferon-dependent innate immunity. Hepatology. 2013;57:46-58.

36. Ding Q, Cao X, Lu J, et al. Hepatitis C virus NS4B blocks the interaction of STING and TBK1 to evade host innate immunity. J Hepatol. 2013;59:52-8.

37. Ono C, Ninomiya A, Yamamoto $\mathrm{S}$, et al. Innate immune response induced by baculovirus attenuates transgene expression in mammalian cells. J Virol. 2014;88:2157-67.

38. Farrell GC, Larter CZ. Nonalcoholic fatty liver disease: from steatosis to cirrhosis. Hepatology. 2006;43:S99-s112.

39. Garcia-Martinez I, Santoro N, Chen Y, et al. Hepatocyte mitochondrial DNA drives nonalcoholic steatohepatitis by activation of TLR9. J Clin Invest. 2016;126:859-64.

40. Ahn J, Xia T, Rabasa Capote A, et al. Extrinsic phagocyte-dependent STING signaling dictates the immunogenicity of dying cells. Cancer Cell. 2018;33:862-873.e865.

41. Lebeaupin C, Vallée D, Hazari Y, et al. Endoplasmic reticulum stress signalling and the pathogenesis of non-alcoholic fatty liver disease. J Hepatol. 2018;69:927-47.

42. Luo X, Li H, Ma L, et al. Expression of STING is increased in liver tissues from patients with NAFLD and promotes macrophage-mediated hepatic inflammation and fibrosis in mice. Gastroenterology. 2018;155:1971-1984.e1974.

43. Yu Y, Liu Y, An W, et al. STING-mediated inflammation in Kupffer cells contributes to progression of nonalcoholic steatohepatitis. J Clin Invest. 2019;129:546-55.

44. Qiao JT, Cui C, Qing L, et al. Activation of the STING-IRF3 pathway promotes hepatocyte inflammation, apoptosis and induces metabolic disorders in nonalcoholic fatty liver disease. Metabolism. 2018;81:13-24.

45. Wang X, Rao H, Zhao J, et al. STING expression in monocytederived macrophages is associated with the progression of liver inflammation and fibrosis in patients with nonalcoholic fatty liver disease. Lab Invest. 2020;100:542-52.

46. Cho CS, Park HW, Ho A, et al. Lipotoxicity induces hepatic protein inclusions through TANK binding kinase 1-mediated p62/ sequestosome 1 phosphorylation. Hepatology. 2018;68:1331-46.

47. Maher JJ. Macrophages steal STING from the infectious disease playbook to promote nonalcoholic fatty liver disease. Gastroenterology. 2018;155:1687-8.

48. Pawaria S, Nündel K, Gao KM, et al. Role of interferon- $\gamma$-producing Th1 cells in a murine model of type I interferon-independent autoinflammation resulting from DNase II deficiency. Arthritis Rheumatol. 2020;72:359-70.

49. Iracheta-Vellve A, Petrasek J, Gyongyosi B, et al. Endoplasmic reticulum stress-induced hepatocellular death pathways mediate liver injury and fibrosis via stimulator of interferon genes. J Biol Chem. 2016;291:26794-805.

50. de Martel C, Maucort-Boulch D, Plummer M, et al. World-wide relative contribution of hepatitis $\mathrm{B}$ and $\mathrm{C}$ viruses in hepatocellular carcinoma. Hepatology. 2015;62:1190-200.

51. Dou Z, Xu C, Donahue G, et al. Autophagy mediates degradation of nuclear lamina. Nature. 2015;527:105-9.

52. Li A, Yi M, Qin S, et al. Activating cGAS-STING pathway for the optimal effect of cancer immunotherapy. J Hematol Oncol. 2019;12:35.

53. Zhu Y, An X, Zhang X, et al. STING: a master regulator in the cancer-immunity cycle. Mol Cancer. 2019;18:152.

54. Harlin H, Meng Y, Peterson AC, et al. Chemokine expression in melanoma metastases associated with $\mathrm{CD} 8+\mathrm{T}$-cell recruitment. Cancer Res. 2009;69:3077-85.

55. Chien Y, Scuoppo C, Wang X, et al. Control of the senescenceassociated secretory phenotype by NF- $\mathrm{KB}$ promotes senescence and enhances chemosensitivity. Genes Dev. 2011;25:2125-36.

56. Kang TW, Yevsa T, Woller N, et al. Senescence surveillance of pre-malignant hepatocytes limits liver cancer development. Nature. 2011;479:547-51.

57. Dou Z, Ghosh K, Vizioli MG, et al. Cytoplasmic chromatin triggers inflammation in senescence and cancer. Nature. 2017;550:402-6.

58. Prabakaran T, Bodda C, Krapp C, et al. Attenuation of cGASSTING signaling is mediated by a p62/SQSTM1-dependent autophagy pathway activated by TBK1. Embo J. 2018;37:e97858.

59. Garcia-Diaz A, Shin DS, Moreno BH, et al. Interferon receptor signaling pathways regulating PD-L1 and PD-L2 expression. Cell Rep. 2017;19:1189-201.

60. Morimoto Y, Kishida T, Kotani SI, et al. Interferon- $\beta$ signal may up-regulate PD-L1 expression through IRF9-dependent and independent pathways in lung cancer cells. Biochem Biophys Res Commun. 2018;507:330-6.

61. Erdal E, Haider S, Rehwinkel J, et al. A prosurvival DNA damage-induced cytoplasmic interferon response is mediated by end resection factors and is limited by Trex1. Genes Dev. 2017:31:353-69.

62. Yoshimoto S, Loo TM, Atarashi K, et al. Obesity-induced gut microbial metabolite promotes liver cancer through senescence secretome. Nature. 2013;499:97-101.

63. Larkin B, Ilyukha V, Sorokin M, et al. Cutting edge: activation of STING in T cells induces type I IFN responses and cell death. J Immunol. 2017;199:397-402.

64. Huang KW, Hsu FF, Qiu JT, et al. Highly efficient and tumorselective nanoparticles for dual-targeted immunogene therapy against cancer. Sci Adv. 2020;6:eaax5032.

65. Tan YS, Sansanaphongpricha K, Xie Y, et al. Mitigating SOX2potentiated immune escape of head and neck squamous cell carcinoma with a STING-inducing nanosatellite vaccine. Clin Cancer Res. 2018;24:4242-55. 
66. Ager CR, Reilley MJ, Nicholas C, et al. Intratumoral STING activation with $\mathrm{T}$-cell checkpoint modulation generates systemic antitumor immunity. Cancer Immunol Res. 2017;5:676-84.

67. Li T, Cheng H, Yuan H, et al. Antitumor activity of cGAMP via stimulation of cGAS-cGAMP-STING-IRF3 mediated innate immune response. Sci Rep. 2016;6:19049.

68. Petrasek J, Iracheta-Vellve A, Csak T, et al. STING-IRF3 pathway links endoplasmic reticulum stress with hepatocyte apoptosis in early alcoholic liver disease. Proc Natl Acad Sci USA. 2013;110:16544-9.

69. Du S, Chen G, Yuan B, et al. DNA sensing and associated type 1 interferon signaling contributes to progression of radiation-induced liver injury. Cell Mol Immunol. 2020. https://doi.org/10. 1038/s41423-020-0395-x.

70. Zhong W, Rao Z, Rao J, et al. Aging aggravated liver ischemia and reperfusion injury by promoting STING-mediated NLRP3 activation in macrophages. Aging Cell. 2020;19:e13186.

71. Thomsen MK, Skouboe MK, Boularan C, et al. The cGASSTING pathway is a therapeutic target in a preclinical model of hepatocellular carcinoma. Oncogene. 2020;39:1652-64.

72. Tang CH, Zundell JA, Ranatunga S, et al. Agonist-mediated activation of STING induces apoptosis in malignant B cells. Cancer Res. 2016;76:2137-52.

73. Cavlar T, Deimling T, Ablasser A, et al. Species-specific detection of the antiviral small-molecule compound CMA by STING. Embo j. 2013;32:1440-50.

74. Conlon J, Burdette DL, Sharma S, et al. Mouse, but not human STING, binds and signals in response to the vascular disrupting agent 5,6-dimethylxanthenone-4-acetic acid. J Immunol. 2013;190:5216-25.

75. Banerjee M, Middya S, Shrivastava R, et al. G10 is a direct activator of human STING. PLoS ONE. 2020;15:e0237743.

76. Zhang X, Liu B, Tang L, et al. Discovery and mechanistic study of a novel human-stimulator-of-interferon-genes agonist. ACS Infect Dis. 2019;5:1139-49.
77. Liu B, Tang L, Zhang X, et al. A cell-based high throughput screening assay for the discovery of cGAS-STING pathway agonists. Antiviral Res. 2017;147:37-46.

78. Wehbe M, Wang-Bishop L, Becker KW, et al. Nanoparticle delivery improves the pharmacokinetic properties of cyclic dinucleotide STING agonists to open a therapeutic window for intravenous administration. J Control Release. 2020;330:1118.

79. Landi A, Law J, Hockman D, et al. Superior immunogenicity of $\mathrm{HCV}$ envelope glycoproteins when adjuvanted with cyclic-diAMP, a STING activator or archaeosomes. Vaccine. 2017;35:6949-56.

80. Lu T, Hu F, Yue H, et al. The incorporation of cationic property and immunopotentiator in poly (lactic acid) microparticles promoted the immune response against chronic hepatitis B. J Control Release. 2020;321:576-88.

81. Guo F, Han Y, Zhao X, et al. STING agonists induce an innate antiviral immune response against hepatitis B virus. Antimicrob Agents Chemother. 2015;59:1273-81.

82. Srikanth S, Woo JS, Wu B, et al. The $\mathrm{Ca}(2+)$ sensor STIM1 regulates the type $\mathrm{I}$ interferon response by retaining the signaling adaptor STING at the endoplasmic reticulum. Nat Immunol. 2019;20:152-62.

83. Haag SM, Gulen MF, Reymond L, et al. Targeting STING with covalent small-molecule inhibitors. Nature. 2018;559:269-73.

84. Li YN, Su Y. Remdesivir attenuates high fat diet (HFD)-induced NAFLD by regulating hepatocyte dyslipidemia and inflammation via the suppression of STING. Biochem Biophys Res Commun. 2020;526:381-8.

Publisher's Note Springer Nature remains neutral with regard to jurisdictional claims in published maps and institutional affiliations. 